\title{
La gestión de crisis online 2008-2018 y su tratamiento en áreas del conocimiento y la comunicación en España
}

\section{Online crisis management and its informational treatment in areas of knowledge and communication in Spain in the period of 2008-2018}

Recibido: 16/12/2019

Aceptado: 06/04/2020

Publicado: 26/06/2020
Emilia Smolak Lozano

esmolaklozano@uma.es https://orcid.org/0000-0001-8193-8786

Damián García Ponce cv5224601491@uma.es https://orcid.org/0000-0003-1733-0973

Universidad de Málaga (España)

Resumen: Debido al auge de Social Media, la gestión de crisis de reputación online cada vez cobra mayor importancia en la agenda setting de los medios de comunicación, en la enseñanza de la profesión de dirección de la comunicación, así como en la práctica profesional. La peculiaridad de flujos comunicativos en el ecosistema digital, la velocidad, el alcance y la interactividad de las redes sociales hace que las crisis gestionadas en el entorno digital se caractericen por su gran impacto y su dificultad de contener los efectos no deseados. Requieren un análisis detallado, buena preparación y planes de contingencia que, por su lado, deben ser reflejados en los análisis mediáticos, profesionales y académicos enmarcados como los fallos, las buenas prácticas o las recomendaciones, basadas en la teoría, praxis y análisis de los hechos. Se ha ideado un estudio que cubre tres ámbitos de información sobre los casos de crisis online: "periodístico", "científico" y "profesional". Se han analizado 
10 años de publicaciones en prensa, academia y plataformas especializadas en España sobre la gestión de crisis online, recogiendo los datos sobre el tratamiento y cobertura de estos casos de estudio. Los resultados reflejan un interés limitado en las tres áreas de información por esta temática y poca fundamentación teórico-práctica en los análisis. Por lo general, las conclusiones abordan la necesidad de fomentar los estudios de caso de crisis en Social Media, con un mayor enfoque hacia los contenidos teóricos, praxis profesional y la cobertura más especializada.

Palabras clave: Crisis, Crisis de reputación, Gestión de crisis, Medios de comunicación, Caso de estudio, Tratamiento periodístico, Social media, Crisis online, Gestión de reputación, Hostilidad en Internet.

Abstract: Due to the rise of Social Media, the management of online reputation crises becomes increasingly important in the agenda setting of the media, in the teaching of the profession of communication management, as well as its practice. The peculiarity of communication flows in the digital ecosystem, the speed, scope and interactivity of social networks means that crises managed in the digital environment are characterized by great impact and difficulty in containing unwanted effects. They require a detailed analysis, good preparation and contingency plans, which in turn must be reflected in the media, professional and academic analyzes framed as failures, good practices or recommendations based on the theory, praxis and analysis of the facts. A study has been devised to cover three areas of information on online crisis cases: "journalistic", "scientific" and "professional". 10 years of publications in the press, academia and specialized platforms in Spain on online crisis management have been analyzed, collecting data on the treatment and coverage of these case studies. The results reflect a limited interest in these three information areas in regards to the topic and a limited theoretical-practical foundation of the studies and news. In general, the conclusions address the need to promote the teaching and research on case studies of crisis in Social Media in Spain with a greater focus on theoretical content, professional praxis and more specialized coverage.

Key words: Crisis, Reputation crisis, Crisis management, Mass media, Case study, Press coverage, Social media, Online crisis, Reputation management, Hostility in Internet. 


\section{Introducción}

El presente estudio tiene como objeto observar y contrastar la evolución de la percepción de las crisis en reputación y redes sociales de los últimos diez años, tal como se aplica y refleja en tres ámbitos: $a$ ) en los medios de comunicación, como un sistema de información para el gran público; $b$ ) en la producción científica, como la base de transferencia de conocimiento y c) en el mundo profesional, como la fuente de buenas prácticas.

La importancia de analizar estos tres ámbitos radica en su interrelación mutua y retroalimentaria como fuentes de información, análisis, implementación y evaluación. A pesar de que los objetivos, misión y visión, de cada uno de estos tres ámbitos, son diferentes a priori, su denominador común abarca la información y la educación de sus respectivos segmentos de sociedad.

De este modo, los medios se convierten en la fuente de información referencial y reflejo de la importancia de los acontecimientos en el momento dado a nivel social y económico; la academia como la fuente de aportación teórica y análisis científico y como base de la praxis; y, por último, el sector profesional como la fuente de las recomendaciones prácticas acerca de la gestión e implementación, es decir, la retroalimentación de la praxis de la teoría académica.

\section{Marco teórico}

A partir de la integración en la vida diaria de las redes sociales y la Internet de las cosas, los paradigmas comunicacionales (tanto individuales como corporativos) han ido sufriendo diversas modificaciones y evoluciones, puesto que conforme la sociedad cambia, dichos modelos van revelando esas modificaciones tanto comportamentales como funcionales.

En este marco las crisis de reputación o "crisis de social media" también han modificado su estructura básica. En el mundo offline pre-Internet se consideraba que las crisis, tanto para atenderlas (respuesta) como para preverla (gestión de riesgos potenciales o issues management), tenían un ciclo de vida lineal: la fase preliminar, la fase aguda, la fase crónica y la fase postraumática (Westphalen \& Piñuel, 1993). 
Sin embargo en investigaciones y teorizaciones posteriores llegamos a las teorías integradoras, como la de Jaques $(2007$, p. 150) en las que las crisis, haciéndose eco de la aplicación de la "teoría del caos en la comunicación de crisis" por parte de Murphy (1996) y Seeger (2002) son contempladas como un ciclo no lineal, es decir, un círculo complejo en el que las crisis pasan de una fase a otra y que siempre pueden volver a surgir de la forma en que se vieron anteriormente, ser recordadas y arrojadas a la luz pública, sumadas al histórico de la organización; ya no permanecen enterradas en un olvido mediático. Esto se debe al hecho de que los medios de comunicación, las bases de datos, las noticias y las redes sociales lo "recuerdan todo", en tanto que la información puede quedar obsoleta, pero (tarde o temprano) se la puede recuperar para reexaminarla.

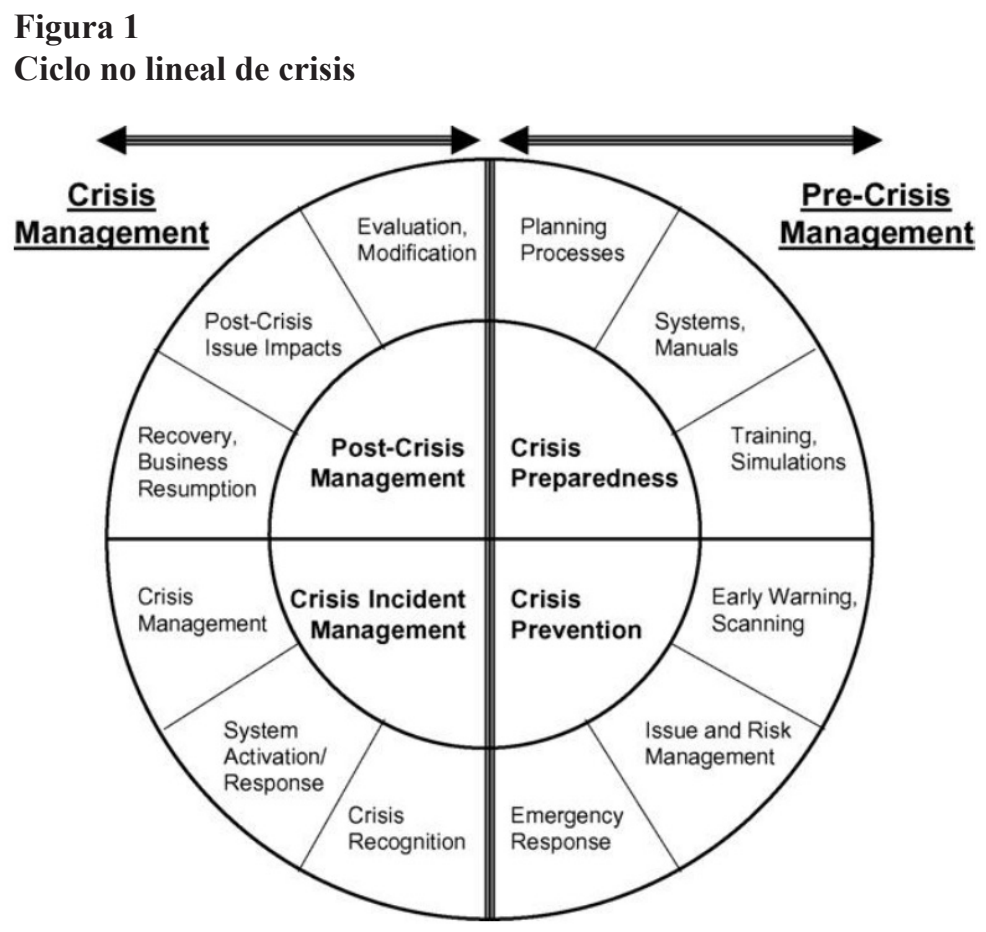

Nota: Jaques (2007, p. 150).

La importancia de estas teorías se debe a que muchas parten de una literatura especializada y que también comparten el estudio de casos para el surgimiento de modelos teóricos como la del "blog mediated crisis communication model" (Jin \& Liu, 2010), que ya parte de crisis originadas en blogs de Internet, 
se habla y profundiza en las "Internet based crises" (Huang, 2006). Es decir, a partir de cierto momento, el mundo online se convierte en el origen y detonante de crisis que afectan a las organizaciones $y$, a través de esta, tocan la reputación de la compañía.

En este panorama surgen teorías y modelos de uso como el "modelo de hostilidad en crisis 2.0" (Castillo \& García, 2015), que prepara un modelo para la identificación, valoración y acción en crisis en redes sociales, partiendo de estudios de caso que permiten sacar los denominadores comunes para la conformación de un modelo así.

En los casos de estudio, las crisis superan el ámbito online muchas veces para llegar a los medios convencionales, pero hay muchas otras crisis que no lo hacen, que permanecen en ese ámbito online donde quedan como una "mancha reputacional", traduciéndose en pérdida de credibilidad, de followers (seguidores) y de alcance para posteriores comunicaciones. Las crisis manejadas por expertos tienen diversos efectos, siendo uno de estos el de la "resiliencia comunicativa" que permite a la marca/organización no verse afectada por ese extremo de desgaste que supone una crisis.

¿Se pierde entonces, en este caso, el factor del histórico como base en el que asentar una estructura de issues management que permita una respuesta meditada en base a lo ya ocurrido en la organización y en otros casos? Podría darse el caso al operar muchas de estas crisis en Internet, donde la información fluctúa a gran velocidad y los históricos pueden quedar enterrados a gran profundidad en las bases de datos.

Rastrear esa información puede ser arduo para el especialista en comunicación. En los casos en los que se pretende confeccionar un histórico es normal recurrir a los medios de comunicación para saber cómo reflejaron información, si la hay, además de haberlo hecho previamente en el histórico de la organización y de los respectivos canales de internet.

El salto de los medios online al offline está mediado por la existencia de un gran número de variables. Liu et al. (2011) establecen dos principales: "social media followers" y "social media inactives", a través de los "social media creators", aparte de la propia organización y sus estructuras y entrega de mensajes. 


\section{Figura 2}

Ciclo de las crisis de social media y flujo de información

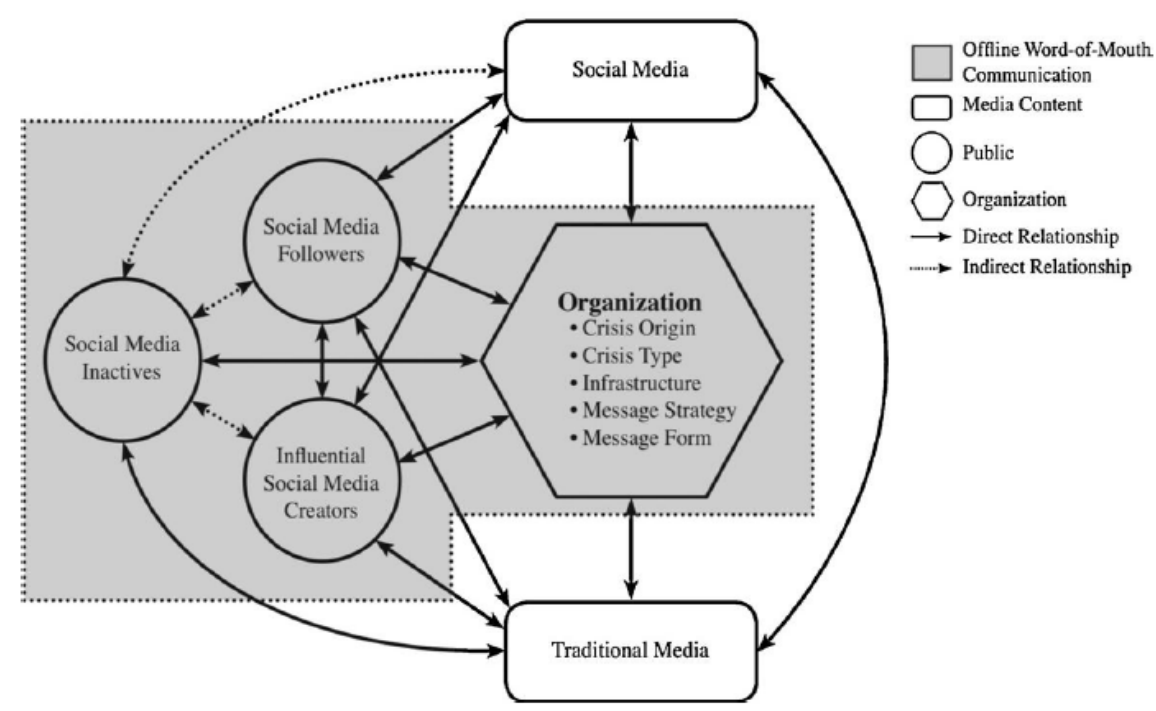

Nota: Liu et al. (2011, p. 347).

La importancia de las crisis (como elementos desestabilizadores del ritmo laboral de una organización y de amenaza a su reputación y estatus en el imaginario colectivo) se representa y configura como algo a lo que prestar atención desde los estadios iniciales donde se pueden detectar esos momentos posibles, que podrían llegar a desembocar en una crisis. Son las llamadas "paracrisis" (Coombs \& Holladay 2012, p. 490) y que se definió también como "elementos de hostilidad comunicativa" (Castillo \& García, 2015).

Las crisis reputacionales muestran una gran posibilidad de incidencia en los asuntos posteriores de la empresa/organización, además de tener influencia en la percepción de la marca y en cómo esta trata sus temas más delicados habiendo o no una crisis de por medio (Weber Shandwick \& KRC Research, 2017). Dado su proliferación y diversidad, una base de histórico de crisis relacionadas con los medios de comunicación masivos y profesionales, así como aportaciones científicas al respecto, parece un recurso clave para el desarrollo científico y profesional en esta vertiente. 
Con esa finalidad, acudimos a sus bases de datos para buscar esas noticias, publicaciones y artículos que han sido determinadas como crisis para las organizaciones. Estamos buscando, como se mencionó anteriormente, una base en la comprobar que las crisis reputacionales o en social media aparezcan reflejadas para realizar ese análisis cuantitativo-cualitativo que nos indique la tendencia en los últimos años.

\section{Metodología}

Partiendo de la perspectiva de una meta-análisis, utilizamos el análisis del contenido y una hermenéutica interpretativa como métodos de investigación. El estudio se centra en territorio español, abarcando los 8 últimos años de los Social Media (2010-2018). El marco temporal responde a la época de la mayor madurez y avance del uso de las redes sociales por parte de las empresas cuando esta práctica empieza a consolidarse a medida que se fortalecen las redes sociales, tales como Facebook, Twitter, YouTube, Instagram, entre otros.

La plantilla de análisis de las fuentes fue diseñada exclusivamente para fines de estudio, con la aplicación especializada a las publicaciones científicas incluidas en las revistas que figuran en Scimago Journal \& Country Rank (SJR, 2019). Como tal, comprende las siguientes variables: enfoques, métodos, temáticas y tipo de crisis; red social, empresa y sector, teorías aplicadas, desarrollo y reacción, efecto final de la crisis.

La herramienta MyNews examinó el impacto mediático del tema en cinco periódicos principales, teniendo en cuenta los factores de tema y empresa en crisis, la cobertura y espacio dedicado. Por último, la monitorización online analizó las publicaciones profesionales de las páginas web especializadas en RR. PP. y marketing digital con más tráfico, basándonos en datos de SimilarWeb y añadiendo variables desde el punto de vista profesional: preparación de planes de contingencia y casos de estudio. 


\section{Figura 3}

Revistas españolas con mayor índice SJR

\begin{tabular}{|c|c|c|c|c|c|c|c|c|c|c|c|}
\hline & Title & Type & $\downarrow S J R$ & $\begin{array}{r}\mathrm{H} \\
\text { index }\end{array}$ & $\begin{array}{r}\text { Total Docs. } \\
\text { (2017) }\end{array}$ & $\begin{array}{r}\text { Total Docs. } \\
\text { (3years) }\end{array}$ & $\begin{array}{l}\text { Total } \\
\text { Refs. }\end{array}$ & $\begin{array}{r}\text { Total Cites } \\
\text { (3years) }\end{array}$ & $\begin{array}{r}\text { Citable Docs. } \\
\text { (3years) }\end{array}$ & $\begin{array}{r}\text { Cites / Doc. } \\
\text { (2years) }\end{array}$ & $\begin{array}{l}\text { Ref./ } \\
\text { Doc. }\end{array}$ \\
\hline 1 & Comunicar $\partial$ & journal & $\begin{array}{r}0.851 \\
01\end{array}$ & 20 & 40 & 124 & 1605 & 307 & 120 & 2.59 & 40.13 \\
\hline 2 & Profesional de la Informacion & journal & $\begin{array}{r}0.652 \\
01\end{array}$ & 20 & 115 & 256 & 4036 & 298 & 256 & 1.25 & 35.10 \\
\hline 3 & Comunicacion y Sociedad & journal & $\frac{0.585}{02}$ & 11 & 50 & 131 & 2112 & 142 & 120 & 0.89 & 42.24 \\
\hline 4 & $\begin{array}{l}\text { Revista Latina de Comunicacion } \\
\text { Social } 2\end{array}$ & journal & $\begin{array}{r}0.571 \\
0.2\end{array}$ & 8 & 82 & 151 & 3412 & 146 & 151 & 0.84 & 41.61 \\
\hline 5 & $\begin{array}{l}\text { Estudios Sobre el Mensaje } \\
\text { Periodistico } \partial\end{array}$ & journal & $\begin{array}{r}0.229 \\
03\end{array}$ & 10 & 89 & 325 & 2969 & 72 & 319 & 0.27 & 33.36 \\
\hline 6 & Historia y Comunicacion Social $\partial$ & journal & $\begin{array}{r}0.218 \\
03\end{array}$ & 5 & 32 & 201 & 1084 & 47 & 197 & 0.27 & 33.88 \\
\hline 7 & Scire & journal & $\begin{array}{r}0.126 \\
04\end{array}$ & 3 & 12 & 68 & 284 & 6 & 68 & 0.09 & 23.67 \\
\hline 8 & $\begin{array}{l}\text { Cultura, Lenguaje y } \\
\text { Representacion } \widehat{\sigma}\end{array}$ & journal & $\begin{array}{r}0.123 \\
Q 4\end{array}$ & 2 & 0 & 43 & 0 & 5 & 39 & 0.26 & 0.00 \\
\hline 9 & Ibersid & journal & $\begin{array}{r}0.116 \\
04\end{array}$ & 1 & 18 & 50 & 489 & 3 & 50 & 0.03 & 27.17 \\
\hline 10 & $\begin{array}{l}\text { IC Revista Cientifica de } \\
\text { Informacion y Comunicacion } 2\end{array}$ & journal & $\begin{array}{r}0.102 \\
Q 44\end{array}$ & 0 & 0 & 10 & 0 & 0 & 10 & 0.00 & 0.00 \\
\hline
\end{tabular}

Nota: Scimago Journal \& Country Rank (SJR, 2019).

La investigación se ha realizado por las siguientes palabras claves: "crisis", "crisis + redes sociales", "redes sociales", "crisis de reputación" y "reputación" en las páginas de las revistas, periódicos y páginas web profesionales dedicadas a la temática. Asimismo, se procedió al análisis de diez revistas españolas de comunicación, según el mayor índice del Ranking SJR en el 2018, registrado en enero-febrero de 2019 (ver Figura 3). El modelo operativo para seleccionar los artículos sobre la temática de la crisis de las empresas y su reputación en redes sociales estuvo basado en una búsqueda por las palabras preestablecidas dentro del buscador en la página web de cada una de las diez revistas. En cuanto al análisis JCR, sólo pudo ingresar la revista Comunicar dentro de las revistas españolas.

A partir de la página de los resultados obtenidos (en términos de volumen de publicaciones desde las palabras claves utilizadas) se ha procedido el análisis cualitativo mediante la ficha especializada de los artículos que respondían al 
perfil establecido como el objeto de estudio. Hay que anotar que Profesional de la Información cuenta con un buscador Google integrado, por lo cual la búsqueda no es tan precisa. En el caso de la Revista Latina de Comunicación Social, sus buscadores (facilitados por la propia página de la revista) presentaban fallos de funcionamiento, por lo cual tuvimos que analizar todos los artículos publicados en dicha revista en el periodo investigado (401 en total). Para extraer estos datos se analizó a los abstracts-resúmenes, la estructura de cada artículo y palabras claves. También para asegurar la viabilidad y exactitud de los datos extraídos se han leído los artículos seleccionados completos.

En cuanto a los medios de comunicación tradicionales, se escogieron los cinco preponderantes por número de lectores y de información general: El País, El Mundo, La Vanguardia, La Voz de Galicia y ABC (Statista, 2019). Aquí hay que destacar que, por limitado uso de metadatos y volumen de producción periodística, hemos visto la necesidad de completar el análisis mediante MyNews con la búsqueda por nombres de las empresas y selección manual de los artículos desde los buscadores integrados dentro las páginas de los periódicos.

Los medios profesionales escogidos son los portales más veteranos y con más visitas, siendo las más populares entre los comunicólogos, los cuales han registrado la mayor cantidad de noticias y que suelen preocuparse por el mundo de la comunicación digital, reflejando los estudios de caso sobre crisis y elementos reputacionales, algunas de las cuales han trascendido a muchos más medios y se han convertido en fuente de referencia para el mundo de la Internet interesado en la meta-investigación de sus propios procesos comunicacionales y una referencia nacional en la práctica empresarial. Así, se analizaron mediante la búsqueda perfilada y plantilla adaptada:

- Marketing Directo.

- PuroMarketing.

- Merca 2.0 (aporta el punto de vista internacional).

\section{Resultados}

A partir de las palabras clave utilizadas se sostiene que las noticias sobre crisis en redes sociales, que atañen a empresas o personas (branding personal), 
son muchas menos de las que cabría esperar por parte de los medios generalistas y algunas más en los medios especializados.

Las bases de datos de los medios generalistas no especializan ni perfilan la información de crisis en redes sociales, donde en muchos casos se tratan más como una anécdota, salvo cuando se trata de titanes empresariales como Facebook o US Airways, donde sí hacen constar un seguimiento del tema. Para investigar los casos en sí, no podemos valernos de palabras clave generales, sino ir al nombre concreto de las crisis o de los que han intervenido en estas.

En nuestro punto de partida queríamos comprobar la validez de la afirmación en la que se sostiene que (dados los años de desarrollo de la comunicación en redes sociales) encontraríamos que los casos de crisis reputacional y en redes sociales decrece con el tiempo, conforme nos acercamos al tiempo presente y hallamos una mayor concienciación por parte de los profesionales. Sin embargo, hemos encontrado una baja permeabilidad de los medios de comunicación para buscar o reflejar esos casos de estudio en sus planas, hallando además un bajo interés e impacto de las crisis reputacionales y una peor nomenclatura de las mismas en sus bases recurribles, entorpeciendo así la investigación.

A esto debemos añadir un escaso interés académico y aportaciones en formato de caso de estudio que podría facilitar la formación a los futuros profesionales en este campo, a la par que también esa baja permeabilidad o estanqueidad se da en los medios profesionales con respecto al mundo académico, que permitiría realizar (con sus investigaciones y avances) mejores análisis útiles para el profesional.

En el ámbito académico, el panorama arroja que los estudios sobre crisis particulares o sobre elementos de crisis reputacional sigue siendo limitado (nunca ha sido algo demasiado generalista) a la luz de los resultados.

\subsection{Las revistas de investigación académica}

El análisis presenta los siguientes valores numéricos en cuanto a los resultados de búsqueda y los valores de la muestra (ver Tabla 1): 
Tabla 1

Resultados de búsqueda por palabras claves y revista

\begin{tabular}{|c|c|c|c|}
\hline Revista & $\begin{array}{l}\text { Número total de } \\
\text { los resultados }\end{array}$ & $\begin{array}{l}\text { Número de los } \\
\text { artículos } \\
\text { estudiados }\end{array}$ & $\begin{array}{l}\text { Porcentaje de } \\
\text { artículo sobre } \\
\text { crisis empre- } \\
\text { sariales en los } \\
\text { resultados de } \\
\text { búsqueda }\end{array}$ \\
\hline \multirow{3}{*}{ Comunicar } & Total: 29 & \multirow{3}{*}{1} & \multirow{3}{*}{$3.44 \%$} \\
\hline & Crisis: 4 & & \\
\hline & $\begin{array}{l}\text { Redes sociales: } \\
25\end{array}$ & & \\
\hline \multirow{3}{*}{$\begin{array}{l}\text { Profesional } \\
\text { de Información }\end{array}$} & Total: 541 & \multirow{3}{*}{1} & \multirow{3}{*}{$0.18 \%$} \\
\hline & Crisis: 533 & & \\
\hline & $\begin{array}{l}\text { Crisis y redes } \\
\text { sociales: } 8\end{array}$ & & \\
\hline \multirow{2}{*}{$\begin{array}{l}\text { Comunicación y } \\
\text { Sociedad }\end{array}$} & Total: 14 & \multirow{2}{*}{1} & \multirow{2}{*}{$7 \%$} \\
\hline & Crisis y redes: 1 & & \\
\hline \multirow[b]{2}{*}{$\begin{array}{l}\text { Revista Latina de } \\
\text { Comunicación }\end{array}$} & Total: 401 & \multirow[b]{2}{*}{3} & \multirow[b]{2}{*}{$0.75 \%$} \\
\hline & Crisis: 5 & & \\
\hline \multirow{2}{*}{$\begin{array}{l}\text { Estudios sobre el } \\
\text { mensaje periodístico }\end{array}$} & Total: 298 & \multirow[b]{2}{*}{3} & \multirow[b]{2}{*}{$1 \%$} \\
\hline & $\begin{array}{l}\text { Crisis y redes } \\
\text { sociales: } 3\end{array}$ & & \\
\hline \multirow{2}{*}{$\begin{array}{l}\text { Historia y } \\
\text { Comunicación Social }\end{array}$} & Total: 229 & \multirow{2}{*}{2} & \multirow{2}{*}{$0.9 \%$} \\
\hline & Crisis: 3 & & \\
\hline \multirow{2}{*}{ Scire } & Total: 3 & \multirow{2}{*}{1} & \multirow{2}{*}{$33.3 \%$} \\
\hline & Crisis: 1 & & \\
\hline $\begin{array}{l}\text { Cultura, Lenguaje y } \\
\text { Representación }\end{array}$ & Total: 0 & 0 & $0 \%$ \\
\hline Ibersid & Total: 0 & 0 & $0 \%$ \\
\hline $\begin{array}{l}\text { IC Revista Científica } \\
\text { de Información y } \\
\text { Comunicación }\end{array}$ & Total: 0 & 0 & $0 \%$ \\
\hline
\end{tabular}

Nota: Elaboración propia.

De totalidad de los resultados, sólo 12 artículos publicados desde 2008-2018 fueron identificados y susceptibles al análisis al ajustarse al tema investigado (ver Tabla 2). El interés en la gestión de crisis en las redes sociales es escaso en las revistas Scopus en España. Las 3 revistas (Cultura, Lenguaje y Representación, Ibersid e IC) no publican nada al respecto. Con respecto al resto, Comunicar es la revista con el mayor índice: sólo publicó un artículo, al igual 
que EPI y Sociedad y Comunicación. Por su parte, Estudios sobre el mensaje periodístico, Revista Latina de Comunicación Social e Historia y Comunicación Social publicaron respectivamente 3, 3 y 2 artículos en los ocho años que comprende el análisis.

Sin embargo, si comparamos estos números con el volumen de publicación, como sucede en el caso de la Revista Latina de Comunicación Social (con 401 artículos), se puede observar que la gestión de la crisis y redes sociales apenas representa el $0,75 \%$ (3 artículos) del total de publicación (401) en ocho años de desarrollo de la investigación científica. A pesar del limitado interés en la temática, podemos observar que tiene tendencia de crecimiento (ver Tabla 1), a medida que avanza la práctica comunicativa de las empresas basada en la implementación de las redes sociales.

Tabla 2

Resultados de búsqueda por palabras claves y revista

\begin{tabular}{|c|c|c|c|c|c|c|c|c|c|c|}
\hline Enfoque & Temática & $\begin{array}{l}\text { Tipo de } \\
\text { crisis }\end{array}$ & $\begin{array}{l}\text { Red } \\
\text { social }\end{array}$ & Empresa & Sector & $\begin{array}{c}\text { Teorías } \\
\text { aplicadas }\end{array}$ & $\begin{array}{l}\text { Desarrollo / } \\
\text { reacción }\end{array}$ & $\begin{array}{c}\text { Efecto } \\
\text { final }\end{array}$ & Método & Año \\
\hline $\begin{array}{l}\text { Imagen } \\
\text { corporativa }\end{array}$ & $\begin{array}{l}\text { Gestión de } \\
\text { crisis, pla- } \\
\text { nificación } \\
\text { y TIC }\end{array}$ & $\begin{array}{l}\text { Crisis de } \\
\text { la marca }\end{array}$ & $\begin{array}{l}\text { TIC en } \\
\text { general }\end{array}$ & Ninguna & General & $\begin{array}{l}\text { Gestión } \\
\text { de riesgos } \\
\text { y plan de } \\
\text { crisis }\end{array}$ & $\begin{array}{l}\text { TIC como alia- } \\
\text { do o enemigo }\end{array}$ & $\begin{array}{l}\text { Reposicio- } \\
\text { namiento / } \\
\text { fortalecer a } \\
\text { la marca / } \\
\text { crisis como } \\
\text { la oportu- } \\
\text { nidad }\end{array}$ & $\begin{array}{l}\text { Modelo } \\
\text { teórico }\end{array}$ & 2008 \\
\hline $\begin{array}{l}\text { Reputación } \\
\text { corporativa }\end{array}$ & $\begin{array}{l}\text { Reputación } \\
\text { online }\end{array}$ & $\begin{array}{l}\text { Emer- } \\
\text { gente del } \\
\text { contexto } \\
\text { digital }\end{array}$ & $\begin{array}{l}\text { Todas } \\
\text { las } \\
\text { redes }\end{array}$ & Ninguna & General & $\begin{array}{l}\text { Modelo de } \\
\text { análisis de } \\
\text { reputación } \\
\text { online }\end{array}$ & $\begin{array}{l}\text { Acciones } \\
\text { comunicativas } \\
\text { de los prosumi- } \\
\text { dores }\end{array}$ & $\begin{array}{l}\text { Evaluación } \\
\text { del conoci- } \\
\text { miento de } \\
\text { los públicos }\end{array}$ & Cualitativo & 2016 \\
\hline $\begin{array}{l}\text { Crisis } \\
\text { como fac- } \\
\text { tor externo } \\
\text { / aconte- } \\
\text { ci-mientos }\end{array}$ & $\begin{array}{l}\text { Galletas } \\
\text { Fontaneda }\end{array}$ & $\begin{array}{l}\text { La crisis } \\
\text { de la } \\
\text { imagen } \\
\text { corpora- } \\
\text { tiva }\end{array}$ & $\begin{array}{l}\text { Medios } \\
\text { de } \\
\text { comuni- } \\
\text { cación }\end{array}$ & $\begin{array}{l}\text { United } \\
\text { Biscuits }\end{array}$ & Alimentación & $\begin{array}{l}\text { Gestión de } \\
\text { crisis / co- } \\
\text { municación } \\
\text { en situación } \\
\text { en crisis }\end{array}$ & $\begin{array}{l}\text { Desde precrisis } \\
\text { hasta poscrisis } \\
\text { con claras y } \\
\text { conflictivas } \\
\text { posturas de } \\
\text { los públicos } \\
\text { implicados }\end{array}$ & $\begin{array}{l}\text { Apariciones } \\
\text { y noticias } \\
\text { en los } \\
\text { medios de } \\
\text { comuni- } \\
\text { cación; } \\
\text { falta de } \\
\text { prevención } \\
\text { comunica- } \\
\text { tiva }\end{array}$ & $\begin{array}{l}\text { Estudio del } \\
\text { caso }\end{array}$ & 2008 \\
\hline $\begin{array}{l}\text { Campañas } \\
\text { durante } \\
\text { emergen- } \\
\text { cias }\end{array}$ & $\begin{array}{l}\text { Huracán } \\
\text { Mathew }\end{array}$ & $\begin{array}{l}\text { Catás- } \\
\text { trofes } \\
\text { naturales }\end{array}$ & $\begin{array}{l}\text { Face- } \\
\text { book }\end{array}$ & ONG & ONG & $\begin{array}{l}\text { Comu- } \\
\text { nicación } \\
\text { horizontal }\end{array}$ & $\begin{array}{l}\text { Mensajes y sus } \\
\text { contenidos }\end{array}$ & $\begin{array}{l}\text { Interacción } \\
\text { con los } \\
\text { usuarios } \\
\text { de los } \\
\text { Community } \\
\text { Manager }\end{array}$ & Encuesta & 2018 \\
\hline $\begin{array}{l}\text { RR. PP. en } \\
\text { desastres } \\
\text { naturales } \\
\text { como la } \\
\text { estrategia } \\
\text { de comuni- } \\
\text { cación }\end{array}$ & $\begin{array}{l}\text { Sismo en } \\
\text { México } \\
2017\end{array}$ & $\begin{array}{l}\text { Catás- } \\
\text { trofes } \\
\text { naturales }\end{array}$ & $\begin{array}{l}\text { Medios } \\
\text { de } \\
\text { comuni- } \\
\text { ca-ción }\end{array}$ & $\begin{array}{l}\text { Gobierno y } \\
\text { ONG }\end{array}$ & $\begin{array}{l}\text { Estatal y } \\
\text { ONG }\end{array}$ & $\begin{array}{l}\text { Comu- } \\
\text { nicación } \\
\text { de crisis; } \\
\text { issue ma- } \\
\text { nagement; } \\
\text { campañas } \\
\text { de informa- } \\
\text { ción }\end{array}$ & $\begin{array}{l}\text { Gestión estra- } \\
\text { tégica de la } \\
\text { comunicación; } \\
\text { en la estrategia } \\
\text { para enfrentar } \\
\text { la crisis }\end{array}$ & $\begin{array}{l}\text { RR. PP. } \\
\text { son muy } \\
\text { útiles para } \\
\text { enfrentar } \\
\text { un desastre } \\
\text { y resarcir } \\
\text { los daños y } \\
\text { desafíos }\end{array}$ & $\begin{array}{l}\text { Encuesta y } \\
\text { estudio de } \\
\text { caso }\end{array}$ & 2018 \\
\hline $\begin{array}{l}\text { Comuni- } \\
\text { ca-ción de } \\
\text { crisis en } \\
\text { emergen- } \\
\text { cias }\end{array}$ & $\begin{array}{l}\text { Atendados } \\
\text { en Reino } \\
\text { Unido }\end{array}$ & $\begin{array}{l}\text { Emer- } \\
\text { gencias }\end{array}$ & $\begin{array}{l}\text { Medios } \\
\text { online } \\
\text { y redes } \\
\text { sociales }\end{array}$ & $\begin{array}{l}\text { Gobierno y } \\
\text { medios }\end{array}$ & $\begin{array}{l}\text { Gobierno y } \\
\text { medios }\end{array}$ & $\begin{array}{l}\text { Comuni- } \\
\text { cación en } \\
\text { catástrofes }\end{array}$ & $\begin{array}{l}\text { Herramientas } \\
\text { utilizadas du- } \\
\text { rante la crisis }\end{array}$ & $\begin{array}{l}\text { Propues- } \\
\text { ta del } \\
\text { protocolo } \\
2.0 \text { para } \\
\text { gestión de } \\
\text { comuni- } \\
\text { cación de } \\
\text { crisis }\end{array}$ & $\begin{array}{l}\text { Análisis } \\
\text { del conte- } \\
\text { nido }\end{array}$ & 2017 \\
\hline
\end{tabular}




\begin{tabular}{|c|c|c|c|c|c|c|c|c|c|c|}
\hline $\begin{array}{l}\text { La gestión } \\
\text { estratégica } \\
\text { de la co- } \\
\text { municación } \\
\text { en crisis }\end{array}$ & $\begin{array}{l}\text { Profesio- } \\
\text { nales en } \\
\text { comunica- } \\
\text { ción }\end{array}$ & $\begin{array}{l}\text { Cualquier } \\
\text { tipo }\end{array}$ & $\begin{array}{l}\text { Social } \\
\text { media }\end{array}$ & $\begin{array}{l}\text { Agencias } \\
\text { de } \\
\text { comunica- } \\
\text { ción }\end{array}$ & Servicios & $\begin{array}{l}\text { Gestión de } \\
\text { crisis, plan } \\
\text { de comuni- } \\
\text { cación }\end{array}$ & $\begin{array}{l}\text { Incorporación } \\
\text { de las redes } \\
\text { sociales en } \\
\text { los planes de } \\
\text { crisis }\end{array}$ & $\begin{array}{l}\text { Las redes } \\
\text { sociales no } \\
\text { están inte- } \\
\text { gramente } \\
\text { incorpora- } \\
\text { das en las } \\
\text { estrategias } \\
\text { de comuni- } \\
\text { cación en } \\
\text { crisis }\end{array}$ & Delphi & 2018 \\
\hline $\begin{array}{l}\text { Comuni- } \\
\text { ca-ción de } \\
\text { crisis en } \\
\text { política }\end{array}$ & $\begin{array}{l}\text { Crisis de } \\
\text { comunica- } \\
\text { ción }\end{array}$ & $\begin{array}{l}\text { Crisis en } \\
\text { política }\end{array}$ & $\begin{array}{l}\text { Medios } \\
\text { online }\end{array}$ & Políticos & Política & $\begin{array}{l}\text { Teoría de } \\
\text { la repara- } \\
\text { ción de la } \\
\text { imagen }\end{array}$ & $\begin{array}{l}\text { Crisis de } \\
\text { imagen política } \\
\text { y demandas } \\
\text { del perdón }\end{array}$ & $\begin{array}{l}\text { Restau- } \\
\text { ración de } \\
\text { imagen }\end{array}$ & $\begin{array}{l}\text { Estudios } \\
\text { de caso }\end{array}$ & 2016 \\
\hline $\begin{array}{l}\text { Comuni- } \\
\text { ca-ción } \\
\text { de crisis a } \\
\text { través de } \\
\text { las redes } \\
\text { sociales }\end{array}$ & $\begin{array}{l}\text { Social } \\
\text { media en } \\
\text { comuni- } \\
\text { cación de } \\
\text { crisis }\end{array}$ & $\begin{array}{l}\text { Crisis en } \\
\text { empre- } \\
\text { sas }\end{array}$ & $\begin{array}{l}\text { Social } \\
\text { media } \\
\text { y redes } \\
\text { sociales }\end{array}$ & $\begin{array}{l}\text { Empresa } \\
\text { en general }\end{array}$ & Privado & $\begin{array}{l}\text { Comu- } \\
\text { nicación } \\
\text { empresarial, } \\
\text { plan de co- } \\
\text { municación } \\
\text { de crisis }\end{array}$ & $\begin{array}{l}\text { Errores en } \\
\text { aplicar social } \\
\text { media en la } \\
\text { gestión de } \\
\text { crisis }\end{array}$ & $\begin{array}{l}\text { Pautas de } \\
\text { cómo apli- } \\
\text { car social } \\
\text { media en } \\
\text { planes de } \\
\text { comuni- } \\
\text { cación de } \\
\text { crisis }\end{array}$ & $\begin{array}{l}\text { Estudios } \\
\text { de casos }\end{array}$ & 2012 \\
\hline $\begin{array}{l}\text { Crisis de } \\
\text { imagen }\end{array}$ & $\begin{array}{l}\text { Crisis } \\
\text { sanitaria }\end{array}$ & $\begin{array}{l}\text { Crisis de } \\
\text { producto }\end{array}$ & $\begin{array}{l}\text { Medios } \\
\text { de comu- } \\
\text { nicación } \\
\text { y RR. } \\
\text { SS. }\end{array}$ & $\begin{array}{l}\text { Aguas de } \\
\text { Mondariz }\end{array}$ & Agricultura & $\begin{array}{l}\text { Gestión de } \\
\text { marca }\end{array}$ & $\begin{array}{l}\text { Impacto } \\
\text { mediático }\end{array}$ & $\begin{array}{l}\text { La res- } \\
\text { puesta de } \\
\text { las marcas } \\
\text { publi- } \\
\text { citarias } \\
\text { españolas }\end{array}$ & $\begin{array}{l}\text { Estudio de } \\
\text { caso }\end{array}$ & 2013 \\
\hline $\begin{array}{l}\text { Crisis insti- } \\
\text { tucional }\end{array}$ & $\begin{array}{l}\text { RR. PP. } \\
\text { en } \\
\text { Entornos } \\
\text { en crisis }\end{array}$ & $\begin{array}{l}\text { Crisis } \\
\text { institu- } \\
\text { cional / } \\
\text { universi- } \\
\text { dad }\end{array}$ & TIC & $\begin{array}{l}\text { Univer- } \\
\text { sidad } \\
\text { andaluza } \\
\text { pública }\end{array}$ & $\begin{array}{l}\text { Educa- } \\
\text { ción-público }\end{array}$ & $\begin{array}{l}\text { RR. PP. } \\
\text { y comu- } \\
\text { nicación } \\
\text { estratégica } \\
\text { en corpora- } \\
\text { ciones }\end{array}$ & $\begin{array}{l}\text { Gabinetes de } \\
\text { prensa }\end{array}$ & $\begin{array}{l}\text { Incorpora- } \\
\text { ción de TIC } \\
\text { en gestión }\end{array}$ & $\begin{array}{l}\text { Revisión } \\
\text { teórica }\end{array}$ & 2014 \\
\hline $\begin{array}{l}\text { Issue ma- } \\
\text { nagement }\end{array}$ & $\begin{array}{l}\text { Monito- } \\
\text { rización } \\
\text { online }\end{array}$ & $\begin{array}{l}\text { Temas } \\
\text { emergen- } \\
\text { tes }\end{array}$ & Twitter & $\begin{array}{l}\text { Institucio- } \\
\text { nes }\end{array}$ & $\begin{array}{l}\text { Documen- } \\
\text { tación }\end{array}$ & $\begin{array}{l}\text { Gestión y } \\
\text { prevención }\end{array}$ & $\begin{array}{l}\text { Detección de } \\
\text { los temas y su } \\
\text { monitorización }\end{array}$ & $\begin{array}{l}\text { Algoritmo } \\
\text { para la } \\
\text { detección } \\
\text { de temas } \\
\text { emergen- } \\
\text { tes }\end{array}$ & $\begin{array}{l}\text { Análisis } \\
\text { del } \\
\text { contenido }\end{array}$ & 2016 \\
\hline
\end{tabular}

Nota: Elaboración propia.

Los datos demuestran grandes lagunas en la investigación comprendida en el ranking Scopus dentro de las mejores diez revistas. Las publicaciones empiezan a crecer a partir del año 2016, sobre todo en 2018 cuando se observa más análisis al respecto. Las redes sociales, sin embargo, ocupan solo una parte limitada y sólo en dos casos se trata de social media en general, así como en otros dos de Facebook y Twitter. El enfoque principal es la crisis de imagen y reputación, crisis de marca en general y crisis en caso de catástrofes y emergencias-comunicación de crisis. Las RR. PP. protagonizan las perspectivas teóricas con la aplicación del corpus de gestión estratégica: la planificación de crisis y su gestión en primer lugar.

Del mismo modo, aparecen enfoques innovadores como comunicación dialógica, mensajes e interactividad de stakeholders, pero el impacto mediático prevalece. Sólo en dos casos contamos con empresas y sectores empresariales como ámbito del desarrollo de las crisis concretos. Estos dos casos hacen referencia a la empresa y al producto, por lo cual se ubican dentro del ámbito 
de la comunicación corporativa. El estudio de caso es el método aplicado con más frecuencia. El sector público e institucional es el campo de análisis predominante. Sólo en tres casos se había propuesto los modelos y pautas con las implicaciones prácticas, siendo las consideraciones teóricas más comunes.

Se puede concluir que en las publicaciones Scopus, en caso de las redes sociales, no se utilizan los estudios de casos enfocados en las empresas para estudiar la crisis de forma muy concreta, aplicable al caso y con la visión de las implicaciones profesionales. En definitiva, la gestión de crisis no es tema de interés dominante y la crisis en social media no trasciende el interés académico, reflejándose de manera muy limitada en la producción final. Seguramente, ampliar este ámbito permitiría acercar la ciencia de la comunicación al mundo profesional, utilizando la capacidad de analizar los datos y casos complejos, hacer comparaciones internacionales $\mathrm{y}$, de este modo, transferir el conocimiento a la sociedad y a la economía española en el campo de desarrollo. El enfoque en los estudios de casos permitiría también crear una base considerable para la docencia y fomentar su calidad.

Las redes sociales conforman un campo bastante nuevo, pero con mucho peso en la gestión empresarial. En ese sentido, la academia española y las revistas indexadas en Scopus deben poner más hincapié a este campo con la implementación práctica. Hacen faltan más estudios para crear un paradigma de comunicación en crisis adaptada a las TIC.

\subsection{Diarios de medios generalistas}

En el caso de los periódicos generalistas, los números de artículos dedicados a las palabras clave indicadas como base de la investigación son concluyentes: no hay relevancia de las crisis reputacionales o en redes sociales de los periódicos, excepto que estemos tratando (como hemos dicho anteriormente) de algún "titán" de la industria de los social media o empresa grande con un caso de crisis muy remarcado, ni como artículos de interés ni como crónicas o reportajes que puedan ayudar al público a formar una opinión y encontrar información concreta sobre un caso en un espacio determinado e informativo. Tampoco resultan de ayuda para los profesionales que estén componiendo una fuente de histórico para realizar su trabajo previo a la preparación de un gabinete o plan de crisis. 
Tabla 3

Medios y noticias

\begin{tabular}{|l|c|c|c|}
\hline \multicolumn{1}{|c|}{ Medio } & $\begin{array}{c}\text { Número de } \\
\text { noticias brutas }\end{array}$ & $\begin{array}{c}\text { Búsqueda } \\
\text { refinada }\end{array}$ & $\begin{array}{c}\text { Total } \\
\text { noticias útiles }\end{array}$ \\
\hline El País & 5197 & 380 & 13 \\
\hline El Mundo & 63300 & 622 & 11 \\
\hline ABC & 6341 & 430 & 13 \\
\hline La Vanguardia & 18100 & 106 & 20 \\
\hline La Voz de Galicia & 136000 & 305 & 11 \\
\hline
\end{tabular}

Nota: Elaboración propia.

En las noticias encontradas con las palabras clave, los resultados son numerosos, pero una vez filtrados en pertinencia (búsqueda refinada) y seleccionando las noticias de utilidad sobre esta investigación, encontramos que no alcanzan a ser notorias. Las noticias útiles que hemos encontrado no provienen finalmente de la búsqueda general en su base de datos, sino de una búsqueda ulterior mediante nomenclaturas relacionadas con elementos participantes en determinadas crisis de reputación (ver Tabla 4).

Tabla 4

Casos de crisis en los medios

\begin{tabular}{|l|c|c|c|c|c|}
\hline \multicolumn{1}{|c|}{ Casos } & $\begin{array}{c}\text { EI } \\
\text { País }\end{array}$ & $\begin{array}{c}\text { La } \\
\text { Vanguardia }\end{array}$ & $\boldsymbol{A B C}$ & $\begin{array}{c}\text { EI } \\
\text { Mundo }\end{array}$ & $\begin{array}{c}\text { La Voz } \\
\text { de } \\
\text { Galicia }\end{array}$ \\
\hline $\begin{array}{l}\text { Kitkat / } \\
\text { Greenpeace }\end{array}$ & 0 & 2 & 2 & 1 & 1 \\
\hline $\begin{array}{l}\text { Nutella / } \\
\text { Campaña }\end{array}$ & 0 & 0 & 0 & 0 & 0 \\
\hline $\begin{array}{l}\text { La Noria / } \\
\text { Pablo Herreros }\end{array}$ & 9 & 17 & 9 & 8 & 9 \\
\hline $\begin{array}{l}\text { ING Direct / } \\
\text { 15M }\end{array}$ & 1 & 0 & 0 & 0 & 0 \\
\hline $\begin{array}{l}\text { Air Europa / } \\
\text { silla de ruedas }\end{array}$ & 1 & 0 & 1 & 1 & 1 \\
\hline $\begin{array}{l}\text { Media Markt / } \\
\text { desfile }\end{array}$ & 0 & 1 & 1 & 1 & 0 \\
\hline
\end{tabular}

Nota: Elaboración propia.

Estos datos finales no permiten seleccionar a los medios generalistas como una fuente de datos sobre crisis reputacional en las redes sociales, debido (so- 
bre todo a la falta de una nomenclatura clara) al bajo interés, salvo cuando se ha visto involucrado un medio de comunicación general (caso de "La Noria" / "Pablo Herreros"). En cuanto a la conformación de datos para un histórico, esta queda en lo anecdótico, debido a lo escaso y difícil de encontrarla, si es que no se ha seguido el caso concreto de forma activa, tanto para su estudio o (en su momento) a la contratación de un clipping de prensa que facilitara dicho trabajo de recolección de datos.

\subsection{Medios profesionales}

Los tres medios profesionales consultados arrojan que, con las mismas nomenclaturas, aparece mucha más información en las palabras clave escogidas (ver Tabla 5).

Tabla 5

Medios profesionales

\begin{tabular}{|l|c|c|c|}
\hline \multicolumn{1}{|c|}{ Medio } & Crisis & $\begin{array}{c}\text { Crisis de } \\
\text { reputación }\end{array}$ & $\begin{array}{c}\text { Crisis en redes } \\
\text { sociales }\end{array}$ \\
\hline Marketing Directo & 6174 & 406 & 1126 \\
\hline Puro Marketing & 2326 & 135 & 10 \\
\hline Merca 2.0 & 3950 & 470 & 1440 \\
\hline
\end{tabular}

Nota: Elaboración propia.

En estos resultados recabamos que las noticias totales recogidas identifican y analizan las crisis, pero sin seguir parámetros investigadores: sólo realizan un relato de lo ocurrido, no interpretativo de causas-efectos ni de las estrategias utilizadas más allá de lo visible, simplemente informando. Esas noticias ocupan las redes sociales en gran parte, debido a su importancia en las campañas de marketing y visibilidad de la compañía. Sin embargo, algo que se echa muy en falta a lo largo de la temporalidad observada es la ponderación (en la mayoría de los casos) de las posibles resiliencias y los beneficios de una buena gestión de crisis, salvando unos pocos artículos recopilatorios que apenas tocan una fracción de todas las informaciones que se dan sobre crisis generales y de reputación.

Los casos de estudio de estos medios (que aparecen únicamente en los resultados de "crisis en redes sociales") poseen métricas relativas al aparta- 
do cuantitativo, a falta de interpretación cualitativa con base académica: son opiniones de profesionales sin conexión con la teoría académica, con pocas posibilidades para la interpretación de los datos. Asimismo, se destaca que ninguna de las noticias refleja el uso o la interpretación del uso de planes de contingencia en casos de crisis, crisis de reputación o crisis en redes sociales, que deberían realizarse mediante una investigación mucho más profunda y en conexión directa con los implicados en las mismas, quedándose (de nuevo) en el horizonte de la mera información.

\section{Discusión y conclusiones}

Los resultados de esta investigación (que pretendía ahondar en la fenomenología de las crisis en redes sociales para poder observar su evolución en cuanto a aumento o decrecimiento de las mismas) no son concluyentes para confrontar dicho fenómeno, debido a los siguientes factores:

- Falta de interés mediático: falta de información y análisis serio del impacto de social media sobre las prácticas empresariales y sus estrategias de comunicación que afectan o pueden afectar la percepción del consumidor, dado que los medios tienen su interés económico, además de su propia agenda.

- Falta de interés académico en el tema y en análisis del caso de estudio: en las revistas de impacto no se dispone de corpus y nomenclatura como fuente fiable, comprobado científicamente y resultante de profundo estudio académico. La crisis no forma parte de la academia de forma sustancial y menos aún las redes sociales, por lo cual en la enseñanza no se dispone de casos y no se enseña así.

La carencia de una base académica sólida (con una nomenclatura clara y bien definida) también es importante a la hora de reclamar el espacio determinado de estudio de la materia y ello se refleja en el tratamiento mediático de la misma y también, parcialmente, en el académico. Sufre el campo profesional sin contar con las referencias científicas, mezclando conceptos y clasificaciones. Los alumnos, la industria, el mercado y los profesionales solo disponen de este campo para aprender las prácticas con las consecuencias que conlleva, sin rigor académico. 
Asimismo, no existe una transferencia de conocimiento hacia la sociedad, hacia la práctica profesional, la economía (entendida como el rendimiento de la empresa) y al mundo académico por escaso interés que despierta en las revistas de referencia: estas no aportan suficiente investigación al desarrollo de las prácticas, nomenclaturas y métodos profesionales, sobre la base de los fundamentos académicos y científicos rigurosos, lo que se traduce en una falta de fundamentos para poder importar el aprovechamiento de dicha base de forma útil y clara hacia el profesional, quien (con la práctica de su lado) pueda contrastar la investigación teórica, obtener unos resultados y que la comunidad académica los recoja para poder reinterpretar y ajustar modelos que ayuden a los profesionales a actualizar, de manera cíclica, dichos conocimientos.

Las crisis en los social media es considerado un campo marginal dentro del mundo académico, mediático y profesional. Empero, sorprende dado su impacto comunicativo, social y económico para empresas y consumidores: dada la facilitad de estudio de los casos, puede ser incluido en la enseñanza en el aula, analizándose su rápido desarrollo, los cambios que sufre, el dinamismo en su aplicación, pudiendo ser abordadas como estudios de casos segmentados. De esta forma, las redes sociales han venido a quedarse: su estudio y aplicación es importante y va más allá del simple conocimiento de los perfiles. En realidad, requiere una visión más profunda en estadística, estudio del mercado, del usuario y del comportamiento de los stakeholders y prosumers hacia el beneficio de la marca.

Es cierto que abundan modelos y aportaciones teóricas en academia, muchos de los cuales hemos mencionado aquí como bibliografía y que se han ido adaptando a los modelos actuales de funcionamiento del mundo comunicativo online. En todo caso, una unificación y taxonomía de los mismo también apoyaría al ámbito académico en su clasificación y fundamentación de la búsqueda de nuevos modelos observacionales, así como casos de estudio con los que aprender del comportamiento de los social media, los influencers y la Internet de las Cosas como fuentes actuales de relación entre empresa, marca y personas.

Existe poco interés mediático en los casos que hemos observado por parte de los medios de comunicación convencionales, ya que están muy influidos y matizados por sus agendas y su prisma de visión mediática. Dichos intereses (entre los que se cuentan los publicitarios) impiden observar y anotar, informar antes que interpretar, como se recomienda deontológicamente, a fin 
de registrar los casos de crisis reputacional y así poder encontrar una nomenclatura clara e identificativa (no parcializada) por los mencionados motivos.

A raíz de esta investigación, se presenta una clara y formidable frontera que pasa por desarrollar modelos, investigar hacia ámbitos relacionados con las redes sociales y el comportamiento de los usuarios, además de formalizar y proponer más y mejores teorías y modelos para su aplicación práctica, su enseñanza en alumnos especializados que lleven esos conocimientos sobre la gestión de crisis al mundo de la praxis profesional.

Resaltamos la baja permeabilidad del mundo académico frente a los estudios de caso profesionales realizados en sus propios entornos, en los que se analiza y recogen datos que se podrían utilizar para elaborar nuevas teorías, modelos y formulaciones, a tenor de hechos comprobables por los profesionales y que normalmente no son tenidos en cuenta si no es para estudio de caso o análisis muy particulares. Esto arroja, para nuestra investigación, la frustrante observación de que tampoco han sido muy permeables estos medios profesionales hacia los avances de investigadores académicos que dotarían de una estructura más sólida a sus análisis.

Nuestra investigación subraya la necesidad de realizar estudios complementarios para poder crear herramientas y modelos más útiles al mundo profesional, mediante la retroalimentación de lo académico con base en informaciones profesionales y estudios de caso fundamentados en las teorías ya existentes. De esta forma, se optimizarían las oportunidades y utilidades en un ciclo colaborativo y de retroalimentación con los medios de comunicación.

\section{Fuentes consultadas}

Arpan, L., \& Roskos-Ewoldsen, D. (2005). Stealing Thunder: Analysis of the effects of proactive disclosure of crisis information. Public Relations Review, 31, 425-433.

Bartos, O., \& Wehr, P. (2002). Using Conflict Theory. Cambridge University Press. 
Barquero, J., \& Castillo, A. (2011). Marco teórico y práctico de las Relaciones Públicas. Furtwangen Editores.

Castillo, A., \& García, D. (2015). Comunicación de crisis. Fragua.

Christen, C. (2005). The restructuring and reengineering of AT\&T: Analysis of a public relations crisis using organizational theory. Public Relations Review, 31, 239-251.

Coombs, W. (1995). Choosing the right words: The development of guidelines for the selection of the "appropriate" crisis response strategies. Management Communication Quarterly, 8, 447-476.

Coombs, W. (2001). Teaching the crisis management/communication course. Public Relations Review, 27, 89-101.

Coombs, W. (2004). Impact of Past Crises on Current Crisis Communication: Insights From Situational Crisis Communication Theory. Journal of Business Communications, 41, 265-289.

Coombs, W. (2007a). Crisis management and communications. Institute of Public Relations. http://www.instituteforpr.org/topics/crisis-management-and-communications/

Coombs, W. (2007b). Attribution Theory as a guide for post-crisis communication research. Public Relations Review, 33, 135-139.

Coombs, W., \& Holladay, S. (2002). Helping crisis managers protect reputational assets: Initial tests of the situational crisis communication theory. Management Communication Quarterly, 16, 165-186.

Coombs, W., \& Holladay, S. (2012). The paracrisis: the challenges created by publicly managing crisis prevention. Public Relations Review, 38, 408-435.

Huang, Y. (2006). Crisis situations, communications strategies, and media coverage: A multicase study revisiting the communicative response model. Communications Research, 33(3), 180-205. 
Jaques, T. (2007). Issue management and crisis management: an integrated non-linear, relational construct. Public Relations Review, 33, 147157.

Jin, Y., \& Liu, B. (2010). The Blog-Mediated Crisis Communication Model: Recommendations for Responding to Influential External Blogs. Journal of Public Relations Research, 22(4), 429-455.

Jin, Y., Pang, A., \& Cameron, G. (2012). Toward a publics-driven, emotion-based conceptualization in crisis communication: Unearthing dominant emotions in multi-staged testing of the Integrated Crisis Mapping (ICM) Model. Journal of Public Relations Research, 24(3), 266-298.

Liu, B., Austin, L., \& Jin, Y. (2011). How publics respond to crisis communications strategies: The interplay of information form and source. Public Relations Review, 37, 345-353. https://www.sciencedirect.com/ science/article/abs/pii/S0363811111000956

Martínez-Nicolás, M., \& Saperas-Lapiedra, E. (2011). La investigación sobre Comunicación en España (1998-2007). Análisis de los artículos publicados en revistas científicas. Revista Latina de Comunicación Social, 66. http://www.revistalatinacs.org/11/art/926_Vicalvaro/05_ Nicolas.html

Murphy, P. (1996). Chaos theory as a model for managing issues and crisis. Public Relations Review, 22(2), 95-113.

Ramos, I., Del Pino, C., \& Castelló, A. (2014). Web 2.0 y redes sociales: estudio de las publicaciones científicas en las revistas españolas de comunicación. Historia y Comunicación Social, 19, 577-590.

Scimago Journal \& Country Rank, SJR. (2019). Journal Rankings 2018 Spain (January-February 2019). https://www.scimagojr.com/journalrank.php? category $=3315 \&$ area $=3300 \&$ country $=E S \&$ type $=j$

Seeger, M. (2002). Chaos and crisis: Propositions for a general theory of crisis communication. Public Relations Review, 28(4), 329-337. 
Seeger, M. (2006). Best Practices in Crisis Communication: An Expert Panel Process. Journal of Applied Communication Research, 34(3), 232-244.

Statista (2019). Número de lectores diarios de los principales periódicos españoles en 2019 (en miles de lectores). https://es.statista.com/estadisticas/476795/periodicos-diarios-mas-leidos-en-espana/

Villagra, N., López, B., \& Monfort, A. (2015). The management of intangibles and corporate branding: Has anything changed in the relationship between business and society? Revista Latina de Comunicación Social, 70, 793-812.

Weber Shandwick, \& KRC Research. (2017). The Company behind the Brand II: In Godness We Trust. http://webershandwick.es/wp-content/uploads/2017/02/CBB-report-FINAL.pdf

Westphalen, M., \& Piñuel, J. (1993). La dirección de comunicación. Ediciones El Prado. 